\title{
A Heterozygous Missense hERG Mutation Associated with Early Repolarization Syndrome
}

\author{
Yun-Jiu Cheng Hao Yao Cheng-Cheng Ji Xu-Miao Chen Jun Fan \\ Li-Juan Liu Su-Hua Wu \\ Department of Cardiology, the First Affiliated Hospital, Sun Yat-Sen University, Key Laboratory on \\ Assisted Circulation, NHC, Guangzhou, China
}

\section{Key Words}

Early repolarization syndrome - Early repolarization pattern - KCNH2 - Gene mutation • Sudden cardiac death

\begin{abstract}
Background/Aims: Early repolarization syndrome (ERS) has been recently recognized as early repolarization pattern with idiopathic ventricular fibrillation. However, the genetic background of ERS has not been fully understood. Methods: A Chinese family with sudden cardiac death associated with ERS was investigated. Direct sequencing of ERS susceptibility genes was performed on the proband and family members. Whole-cell patch-clamp methods were used to characterize the mutant channel expressed in HEK 293 cells. Results: One missense mutation (p. K801T) was found in the hERG (KCNH2 gene) by the direct sequencing of candidate genes. Whole cell voltage clamp studies of the K801T mutation in HEK 293 cells demonstrated a 1.5fold increase in maximum steady state current $(37.2 \pm 7.3 \mathrm{vs} 20.3 \pm 4.4 \mathrm{pA} / \mathrm{pF})$ that occurred at a $20 \mathrm{mV}$ more positive potential compared to the wild type channels. The voltage dependence of inactivation was significantly shifted in the positive voltage direction (WT $-59.5 \pm 1.4$ vs $\mathrm{K} 801 \mathrm{~T}-44.3 \pm 1.2 \mathrm{mV}$ ). Kinetic analysis revealed slower inactivation rates of K801T, but faster rates of activation and deactivation. The hERG channel blockers tested inhibited K801T-hERG channel in concentration response, and the potencies of these drugs can be rank-ordered as follows: quinidine> disopyramide > sotalol> flecainide. Conclusion: Our study indicated that the K801T mutation caused the gain of function of hERG channels that may account for the clinical phenotype of ERS. Quinidine and disopyramide could improve the function of K801ThERG mutant channel, and may be therapeutic options for patients with the K801T hERG mutation.




\section{Cellular Physiology Cell Physiol Biochem 2018;51:1301-1312

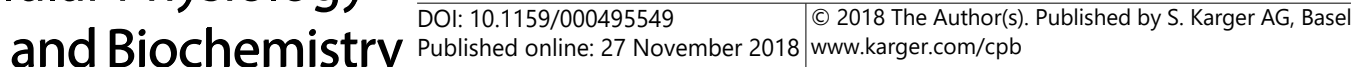 \\ Cheng et al.: A Heterozygous Missense hERG Mutation Associated with Early \\ Repolarization Syndrome}

\section{Introduction}

Early repolarization pattern (ERP) on the electrocardiogram (ECG) is defined as J point elevation $\geq 0.1 \mathrm{mV}$ or QRS notching or slurring in at least 2 contiguous inferior and/or lateral leads [1]. It is prevalent in $2.3 \%$ to $29.3 \%$ of the population depending on race, age and gender category [2,3]. Several recent reports and our previous studies indicated that ERP was associated with increased risk for ventricular fibrillation (VF) and sudden cardiac death (SCD) in the general population and in patients with structural heart disease $[2,4-6]$. The 2015 ESC Guidelines for the management of patients with ventricular arrhythmias defined early repolarization syndrome (ERS) as the presence of ERP with resuscitation from a documented episode of unexplained VF or polymorphic ventricular tachycardia (VT) [7].

The autosomal dominant inheritance nature of the ERP has been suggested by casecontrol studies as well as by studies in the general population, but the familial inheritance of malignant ERS has not been clearly demonstrated [4, 8]. So far, rare genetic variants in genes governing cardiac repolarization, including SCN5A, KCNJ8, and L-type calcium channel subunits, have been shown to be causally associated with ERS [9]. Human hERG (KCNH2 gene) encodes $\alpha$-subunit of the rapid component of the delayed rectifier $\mathrm{K}+$ channel, which plays an important role in regulating the repolarization of the cardiac action potential [10]. In the present study, a Chinese family with the clinical phenotype of ERS was found to have a novel mutation in the hERG gene, K801T, and we report herein that this newly discovered mutation in hERG causes a substantial gain-of-function. We also tested the effects of antiarrhythmic drugs which could prolong cardiac repolarization on the mutant K801T hERG channels expressed in HEK 293 cells.

\section{Materials and Methods}

\section{Clinical investigation}

The proband with a strong family history of sudden cardiac death was referred to us for further evaluation. A complete battery of studies including medical history, physical examination, electrolytes, 12lead ECG, 24-hour Holter monitoring, echocardiography, coronary angiography and genetic testing were done. The study conformed to the principles outlined in the Declaration of Helsinki. This study was approved by the institutional review board, and the patients provided written informed consent for participation.

\section{Genetic analysis}

Genomic DNA was extracted from peripheral lymphocytes using a TIANamp Blood DNA isolation kit (Tiangen, Beijing, China) according to the manufacturer's instructions. All exons of the hERG, KCNE1, KCNE2, SCA5A, KCNJ8, KCNA4, KCND2, KCND3, CACNA1C, CACNB2 and CACNA2D1 genes were amplified by polymerase chain reaction (PCR) and were analyzed by direct sequencing. Sequencing results were compared to 150 healthy subjects of the same ethnic background with normal ECGs to determine the distinction between mutation and polymorphism.

\section{Site-directed mutagenesis and hERG constructs}

Nucleotide change identified in ERS proband was introduced into hERG cDNA cloned in pGFPires as described previously [11]. Human embryonic kidney (HEK) 293 cells were transfected with the WT- or K801T-HERG constructs using an Effectene Transfection Reagent (Qiagen, Hilden, Germany) according to the manufacturer's protocol. The day after transfection, cells were released with trypsin using standard methods and distributed into six well plates, and those cells exhibiting green fluorescence $12-20 \mathrm{~h}$ posttransfection were selected for subsequent electrophysiological study. 


\section{Cellular Physiology Cell Physiol Biochem 2018;51:1301-1312

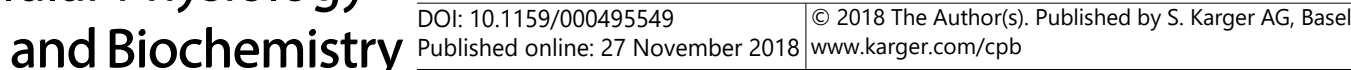 \\ Cheng et al.: A Heterozygous Missense hERG Mutation Associated with Early \\ Repolarization Syndrome}

\section{Electrophysiological analysis in transfected HEK 293 cells}

Functional characterization of WT- and mutant hERG-channels was performed by voltage clamp techniques using transfected HEK 293 cells. Patch clamp recordings were made using low-resistance electrodes ( $<3 \mathrm{MOhm}$ ), and a routine series resistance compensation by an Axopatch 200B amplifier (Axon Instruments Inc, Foster City, CA) was performed to minimize voltage-clamp errors. The patch pipette solution contained (mmol/L): $140 \mathrm{KCl}, 10 \mathrm{NaCl}, 1 \mathrm{MgCl}_{2}, 10 \mathrm{HEPES}$ and 10 EGTA (pH 7.3 with KOH). The extracellular bath solution contained (mmol/L): $140 \mathrm{NaCl}, 5 \mathrm{KCl}, 1 \mathrm{MgCl} 2,2 \mathrm{CaCl} 2,10 \mathrm{HEPES}$ and 10 glucose (pH 7.4 with $\mathrm{NaOH}$ ). Membrane currents were recorded in the whole cell configuration using an Axopatch 200B amplifier controlled by pCLAMP10.3 software (Molecular Devices, Sunnydale, CA). Specific voltageclamp protocols used to determine voltage dependence of activation, inactivation, and recovery from inactivation are illustrated in the figure legend. All experiments were performed at room temperature. Normalized activation and inactivation curves were fitted by a single component Boltzmann model: $\mathrm{I}=\mathrm{I}_{\max } /$ $\left.\left(1+\exp \left[\left(V_{0.5}-V_{m}\right) / k\right)\right]\right)$, where I is the tail current amplitude following test potential $V_{m}, I_{\max }$ is the maximum current density of activation or inactivation, $\mathrm{V}_{0.5}$ is the half-maximum activation or inactivation voltage, and $\mathrm{k}$ is the slope factor for the relationship.

The effects of hERG channel blockers were determined using a two step protocol. hERG potassium currents were elicited by 4-s voltage pulses to $+20 \mathrm{mV}$ from a holding potential of $-80 \mathrm{mV}$, followed by a repolarizing ramp to $-80 \mathrm{mV}$, eliciting a large outward tail current. Steady current at the end of the first step and the peak tail current were determined before and during the response to the blockers. The concentration-response relationship of hERG channel blockers was quantified by fitting the data using the Hill equation: $\mathrm{I}_{\text {drug }} / \mathrm{I}_{\text {control }}=1 /\left[1+\left(\mathrm{D} / \mathrm{IC}_{50}\right)^{\mathrm{p}}\right]$, where $\mathrm{D}$ is the drug concentration, $\mathrm{p}$ is the Hill coefficient, $\mathrm{IC}_{50}$ is the concentration for $50 \%$ inhibition. $\mathrm{IC}_{50 \text {-steady }}$ and $\mathrm{IC}_{50 \text {-tail }}$ are the concentration for $50 \%$ inhibition of steady current and tail current, respectively [12].

\section{Statistical analysis}

Data are represented as the means \pm standard error of the mean (SEM) unless otherwise indicated. Statistical analysis of the data was performed using a Student's t test for paired data or a one-way ANOVA for comparisons of multiple means with statistical software SPSS 19.0. Statistical significance was set at $\mathrm{P}<0.05$.

\section{Results}

\section{Clinical characteristics}

The proband, a 42-year-old male patient was admitted to the First Affiliated Hospital of Sun Yat-Sen University due to a history of recurrent syncope at rest (once or twice every year since 5 years ago). His previous medical records indicated that he often presented with chest discomfort and palpitation, followed by which transient loss of consciousness occurred. The ECG that was obtained at the emergency department exhibited positive QRS complex, J-point elevation by $0.2 \mathrm{mV}$, descending ST segment and negative T waves in inferior (II, III and aVF) leads (Fig. 1A). During the admission process, VT and subsequent VF episode, which were terminated successfully by direct current cardioversion, were documented on the ECG obtained in the ward (Fig. 1B, 1C). There was no difference in clinical state that may explain the marked J waves in ECG. Myocardial infarction was excluded by cardiac enzyme tests and coronary angiography. Structural abnormalities were excluded by echocardiography, and electrolyte disturbances were excluded by laboratory tests. According to the clinical manifestations, he was diagnosed with ERS and received an implantable cardioverter defibrillator implantation.

The family pedigree is presented in Fig. 1D. A total of 9 family members were evaluated clinically and genetically, and four of them suffered SCD (Fig. 1D; I-1, II-1, II-2, II-4) at relatively young ages (35 to 52 years old) all of which occurred at night. Other family members were asymptomatic. In the ECG of the proband's 31-year-old sister (III-2), terminal QRS notches in leads II, III and aVF (J-point elevation $>0.1 \mathrm{mV}$ ) with horizontal ST-segment were observed (Fig. 1E). The ERP ECG pattern with terminal QRS notches or slurrings was also observed in both his younger cousins (Fig. 1E). In addition, ERP phenotype in inferior and lateral leads 
Fig. 1. Family pedigree and representative ECG of ERS patients from a Chinese family. (A) ECG during sinus rhythm showed terminal QRS slurring in leads II, III and aVF (arrows) with ST-segment depression; (B) ECG recorded during admission showed ventricular tachycardia; (C) A spontaneous onset of ventricular fibrillation; (D) Phenotypic traits are represented by specific symbols in the family pedigree; (E) ECG recordings of the proband's sister and two cousins indicate ERP phenotype.

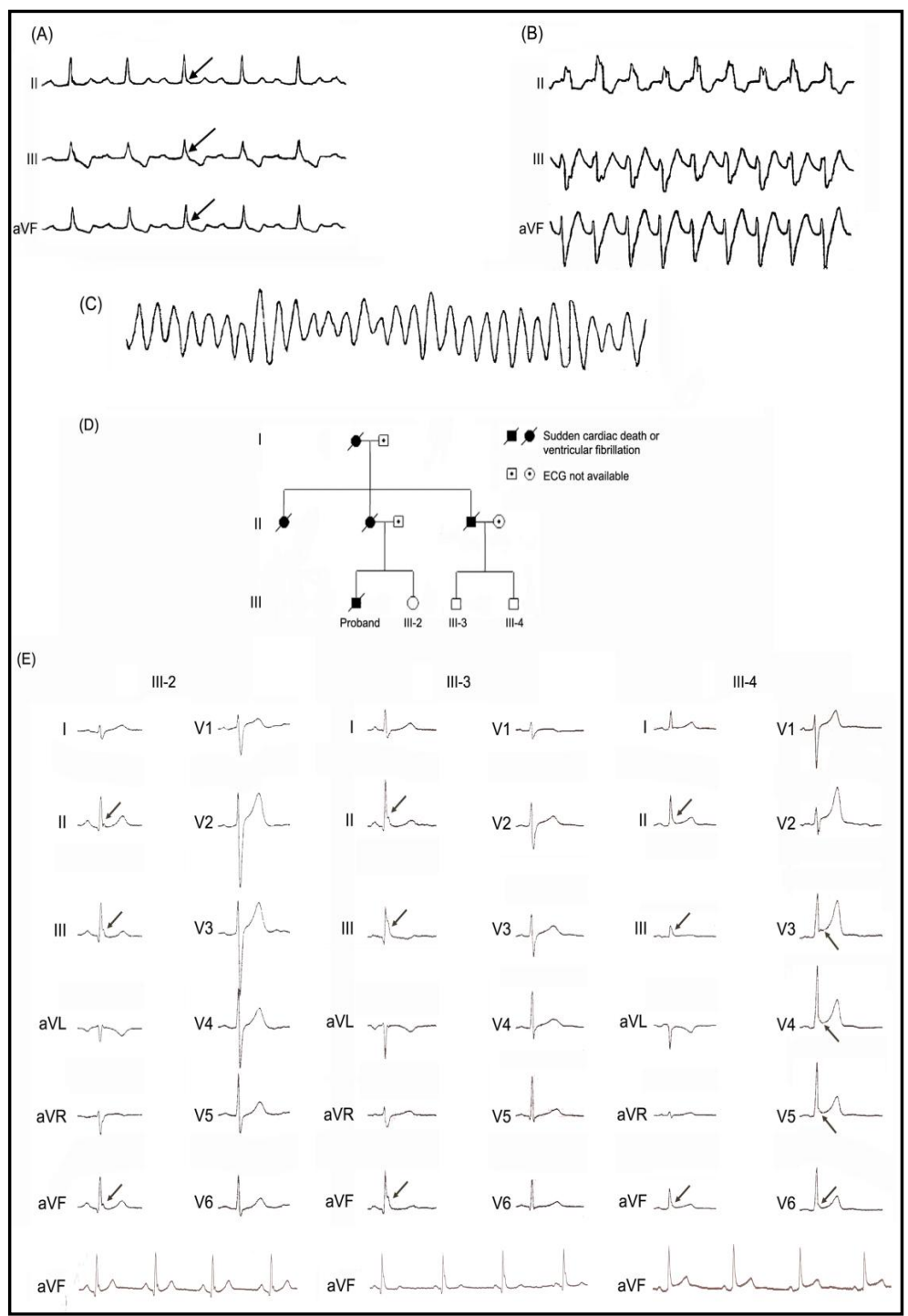

was also observed in the proband's decreased mother (II-2) (Fig. 2). The QTc interval for the family members with ERP were shorter than the ethnically matched controls ( $384 \pm 12 \mathrm{~ms}$ vs. $415 \pm 9 \mathrm{~ms}, \mathrm{p}=0.02$ ).

\section{The result of mutation analysis}

Genetic studies of the patients' DNA excluded mutations in SCN5A, KCNQ1, KCNE1, KCNE2, KCNE3, HCN4, CACNA1C, CACNB2. A single base pair substitution (A to C substitution at nucleotide 2402, c.2402 A>C) was found in the hERG gene of the proband (Fig. 3A), resulting in an amino acid change from lysine to threonine at position 801 (p.K801T) in the hERG potassium channel. The schematic topology in Fig. 3C depicts location of this mutation which is located in C-terminus of hERG protein, and is therefore likely to influence function. The same mutation was present in his younger sister and two cousins. However, this mutation was not observed in the proband's father, indicating the K801T mutation of the proband could have been inherited from his decreased mother. The K801T substitution was not present in any of 150 control subjects of the same ethnic background, and has not been reported according to the NHLBI Exome Sequencing Project, Exome Variant Server (http://evs.gs.washington.edu/EVS/), suggesting that the mutation is associated with the phenotype. 


\section{Cellular Physiology Cell Physiol Biochem 2018;51:1301-1312

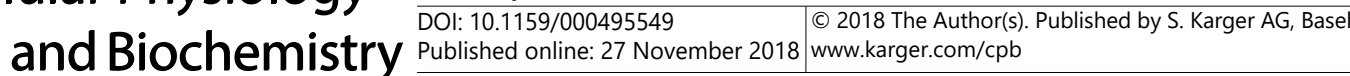

Cellular electrophysiology

To determine the effects of the K801T mutation on hERG channel function and its contribution to the clinical phenotype, we expressed WT- and K801T-hERG constructs in HEK 293 cells for electrophysiological analysis. Whole-cell recordings showed that both WTand K801T-hERG currents elicited by sequential depolarizing pulses exhibit slow initial rates of activation with peak current increasing with voltage to a maximum. With further increases in voltage, the current decreases because of the rapid onset of inactivation (rectification). WT and K801T recordings also displayed the typical large tail currents generated by inactivated channels rapidly reopening on repolarization.

Current from the cells expressing the two hERG constructs was measured at the end of the first voltage clamp step, normalized by cell capacitance and averaged. A comparison of the steady current density-voltage relations between the WT- and K801T-hERG channels is shown in Fig 4B. The peak current occurred at about +10 $\mathrm{mV}$ for WT-hERG and at about $+30 \mathrm{mV}$ for K801ThERG, and was approximately 1.5-fold greater for K801T- $(37.2 \pm 7.3 \mathrm{pA} / \mathrm{pF}, \mathrm{n}=25)$ compared to WT-hERG $(20.3 \pm 4.4 \mathrm{pA} / \mathrm{pF}, \mathrm{n}=18, \mathrm{P}<0.001)$. Fig. 4C shows activation curves derived from tail currents for WT- and K801T-hERG channels,

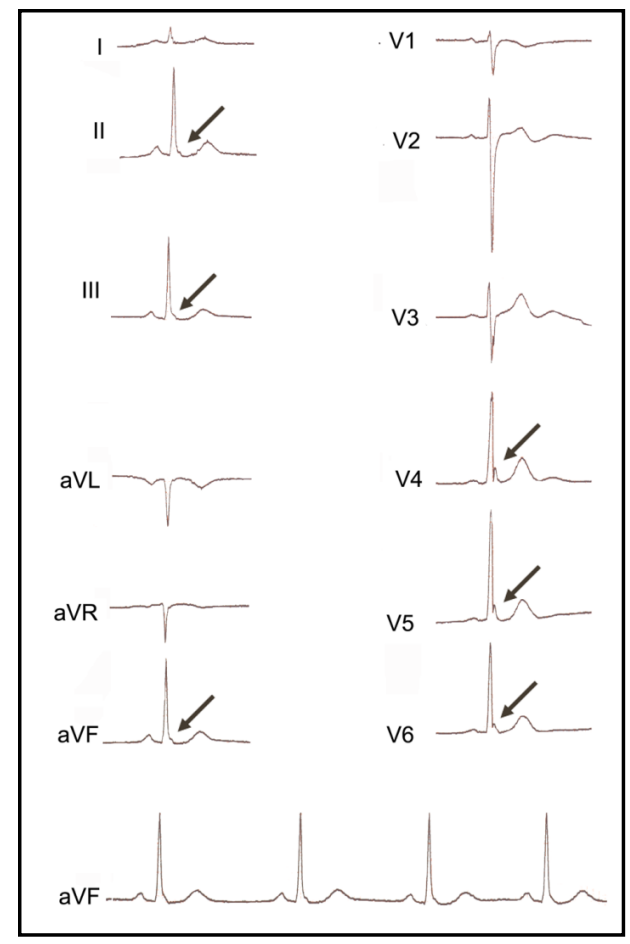

Fig. 2. Representative ECG of the proband's mother who suffered SCD. The ECG before her death showed ERP in leads II, III, aVF and V46.

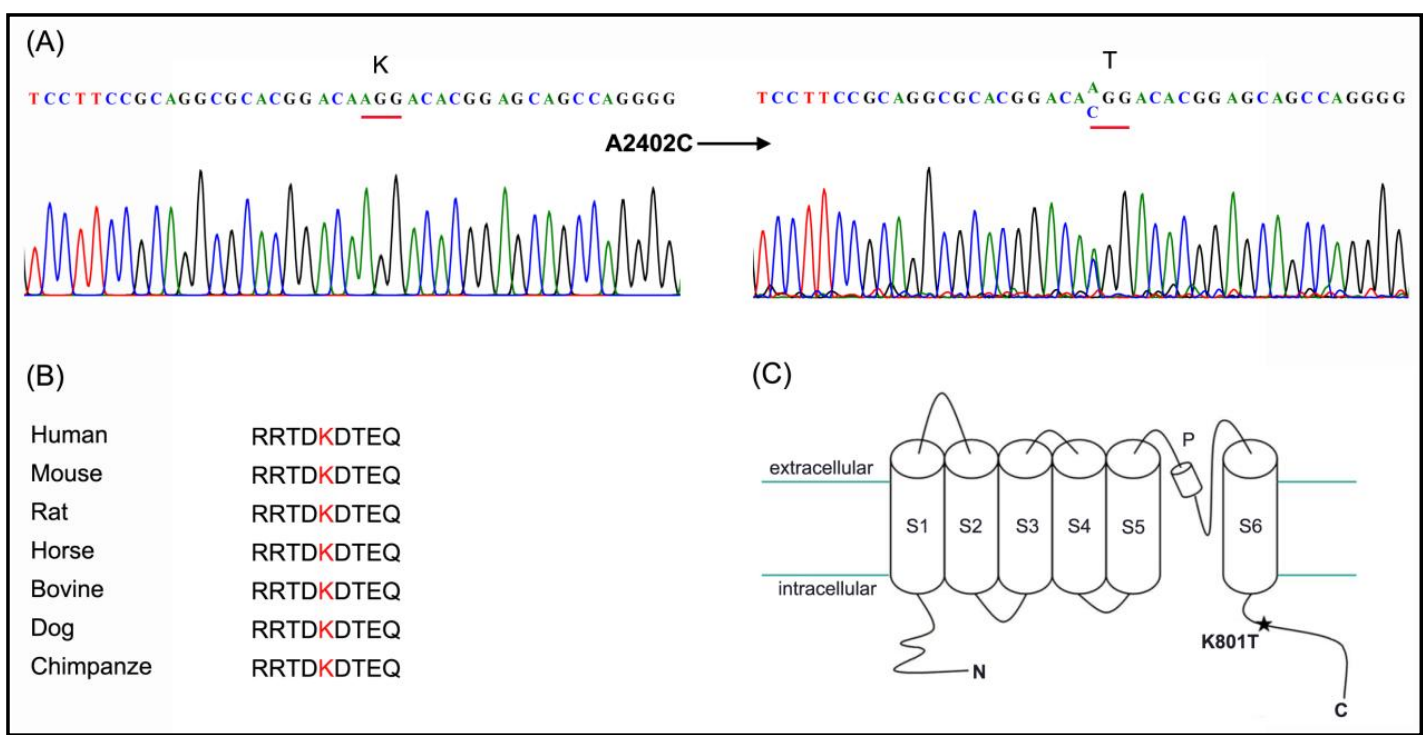

Fig. 3. Variant of hERG gene identified in the family. (A) A missense mutation (A to $C$ at nucleotide 2402) results in the substitution of lysine at codon 801 by threonine (K801T). (B) Amino acid sequence alignment of hERG channel from various species in the region surrounding amino acid 801 (highlighted). The highly conserved sequence suggests functional relevance of this region. (C) A schematic topology showing the hERG channel and locations of the K801T mutation described. $\mathrm{C}=\mathrm{C}$-terminus; $\mathrm{N}=\mathrm{N}$-terminus; $\mathrm{P}=$ pore region; S1-S6 = transmembrane segments. 
Fig. 4. Activation kinetics of hERG current reconstituted by wild type (WT) and K801T. (A) R e pres en t a tive current traces of hERG channel recorded from HEK 293T cells transfected with WT or K801T mutation. (B) Steady current measured at the end of the first voltage step normalized by cell capacitance and averaged at each voltage. (C) Current voltage relation for WT- and K801T-hERG tail current recorded at $-40 \mathrm{mV}$ following repolarization from the test potentials plotted on the membrane potential axis. (D) Measurement of rate of activation of WT- or K801ThERG channels. (E) Plots of time-course of hERG channel activation obtained using an "envelopeof-tails" protocol. For each cell, the peak current amplitudes at each time-point were normalized to the maximal current observed during the

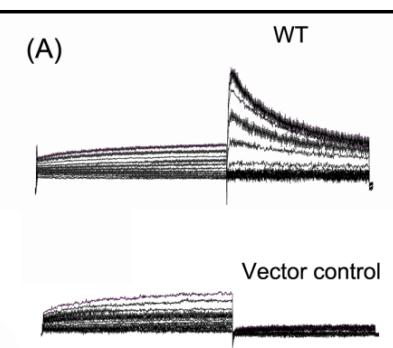

(B)

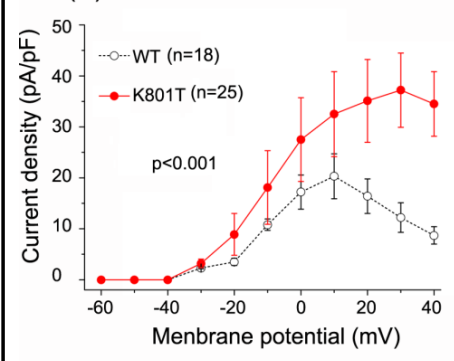

(D)
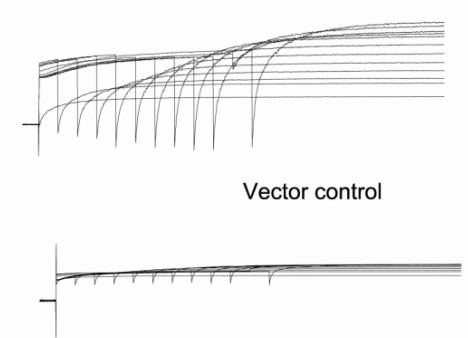
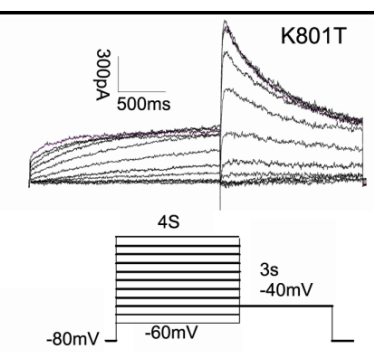

(C)
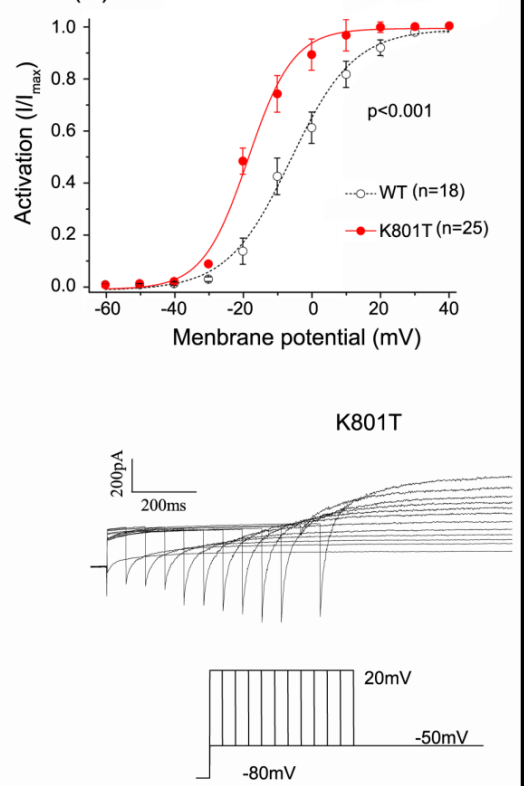

(E)

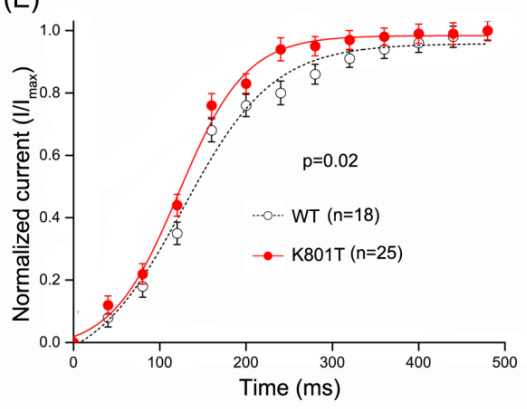
protocol.

fitted with a Boltzmann model. The half-activation voltage of K801T-hERG was $-18.5 \pm 0.9$ $\mathrm{mV}$, whilst for WT-hERG this was $-6.0 \pm 1.2 \mathrm{mV}(\mathrm{P}<0.001)$, indicating a significant voltage shift in the K801T-hERG compared to WT. The corresponding $\mathrm{k}$ values for K801T- and WT-hERG channels were $6.6 \pm 0.8 \mathrm{mV}$ and $9.2 \pm 1.0 \mathrm{mV}$, respectively $(\mathrm{p}<0.001)$. We used an "envelope of tails protocol" to investigate effects of the K801T mutation on the time-course of hERG activation. Mono-exponential fits to the data yielded a $\tau_{\text {activation }}$ of $128.3 \pm 3.5 \mathrm{~ms}$ for WT-hERG and of $121.2 \pm 4.3 \mathrm{~ms}$ for K801T-hERG ( $\mathrm{n}=18$ and 25 cells respectively; $\mathrm{P}=0.02)$, indicating that activation rate for K801T-hERG was faster than WT- hERG during this protocol (Fig. 4E).

Fig. 5A illustrates 2 representative current traces showing the hERG channel deactivation at various test potentials after depolarization to $+60 \mathrm{mV}$. The decaying phases of tail currents could be fit to the sum of 2 exponential equations. The time constant of Tau- 


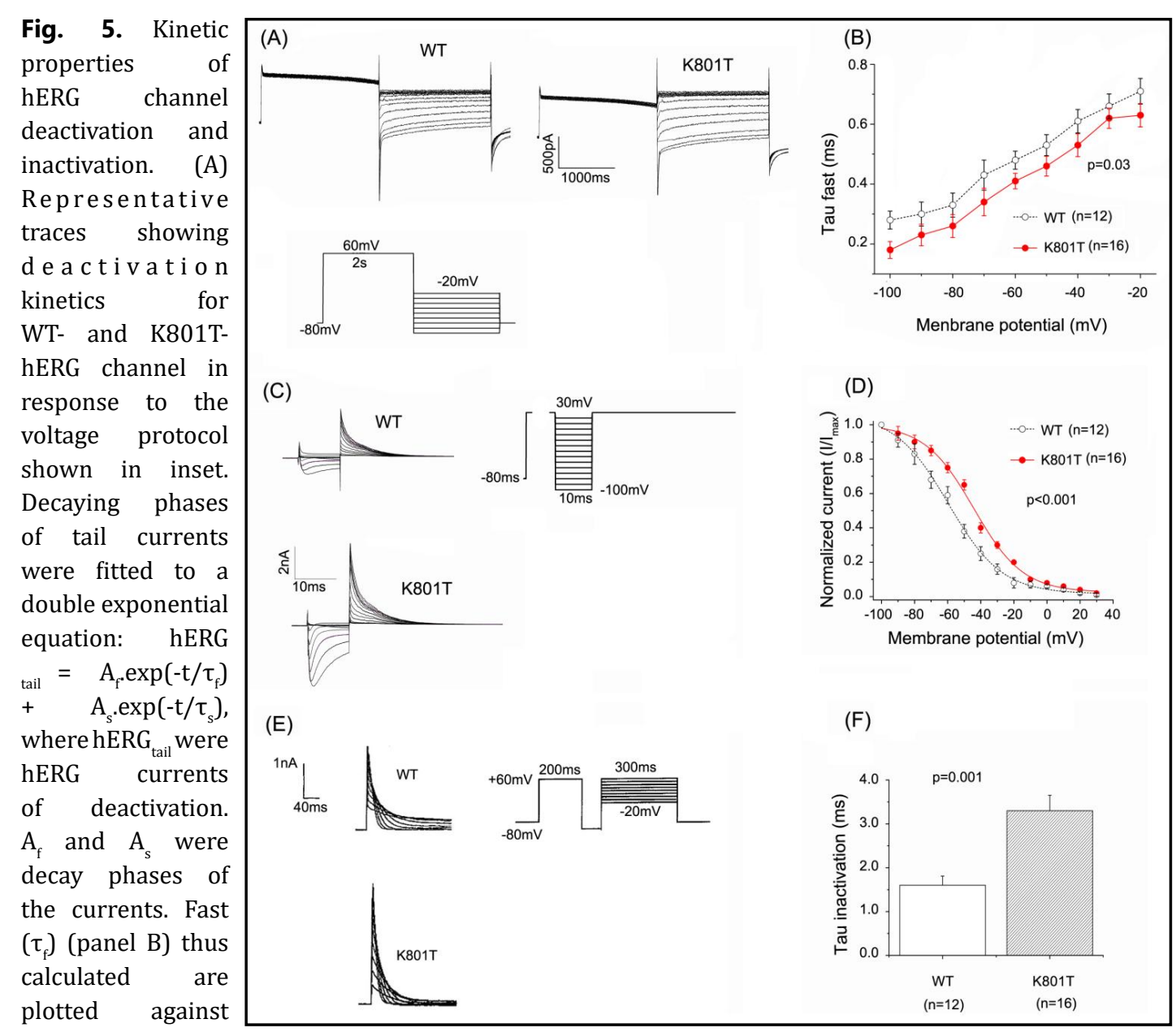

test potentials. (C)

Representative traces showing inactivation kinetics for WT- and K801T-hERG channel in response to the voltage protocol shown in inset. (D) Normalized inactivation curves were fitted by the Boltzmann equation. (E) Representative traces showing time-course of development of inactivation for WT- and K801T-hERG channel in response to the voltage protocol shown in inset. (F) Bar charts comparing time constant of WT and K801-ThERG channel inactivation. Inactivating currents were fitted with a standard mono-exponential function.

fast was thus measured and is plotted against the test potentials in Fig. 5B.The fast phase of deactivation was faster for K801T than WT-hERG ( $p=0.03$ ). Inset for Fig. 4C shows the patchclamp protocol to measure the steady-state voltage dependence of the inactivation gate and experimental results for WT- and K801T-hERG currents. Peak tail current densities recorded at P3 in multiple cells are normalized to the maximal hERG current during the protocol and plotted against test potentials (P2) in panel D. The inactivation $\mathrm{V}_{0.5}$ value for WT-hERG current derived from a fit to the data with Boltzmann equation was $-59.5 \pm 1.4 \mathrm{mV}$ with a $\mathrm{K}$ value of $14.9 \pm 1.2 \mathrm{mV}$ ( $\mathrm{n}=12$ cells). For K801T-hERG current, the corresponding values were: $\mathrm{V}_{0.5}$ of $-44.3 \pm 1.2 \mathrm{mV}$ and $\mathrm{k}$ of $16.2 \pm 1.2 \mathrm{mV}$ ( $\mathrm{n}=16$ cells; and, respectively, $\mathrm{p}<0.001$ and 0.25 versus control) (Fig. 5D). The time-course of development of inactivation for WT and K801ThERG was compared by mono-exponential fitting of the decline of transient currents elicited following the repolarizing step to $-120 \mathrm{mV}$. This yielded $\tau$-values for WT and K801T-hERG inactivation of $1.6 \pm 0.2 \mathrm{~ms}$ and $3.3 \pm 0.4 \mathrm{~ms}$ respectively (Fig. $5 \mathrm{~F} ; \mathrm{n}=12$ and 16 respectively; $\mathrm{p}=0.001$ ). 


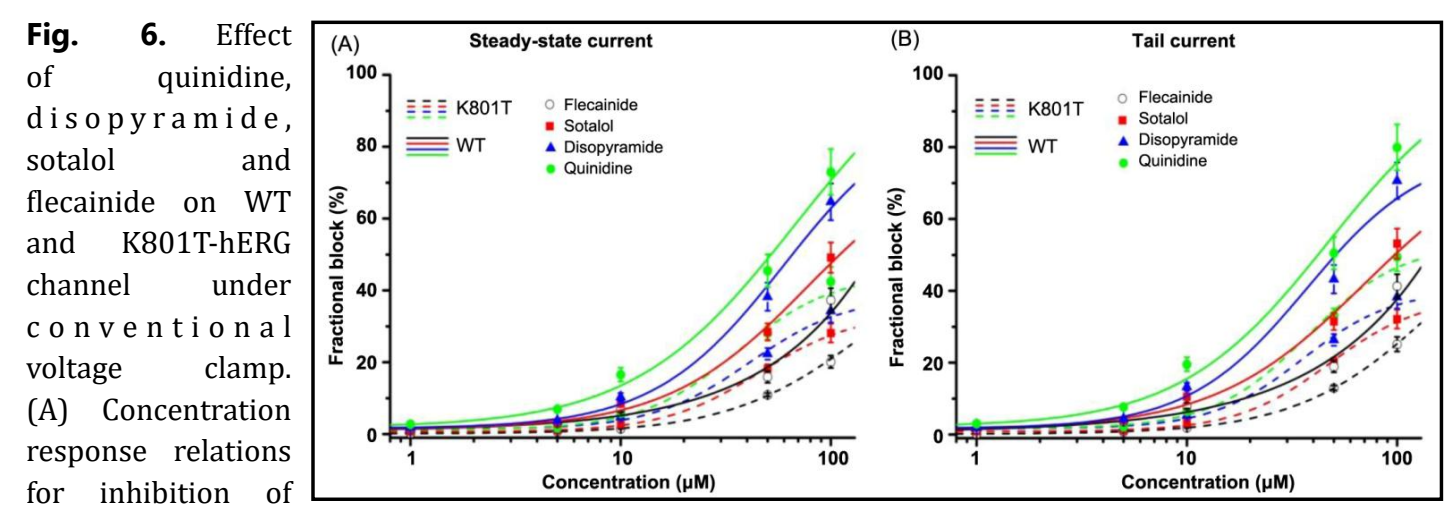

steady state of WT

and K801T-hERG channel by four drugs tested. (B) Concentration response relations for inhibition of tail current of WT and K801T-hERG channel by four drugs tested.

Table 1. Effects of four hERG channel blockers, quinidine, disopyramide, sotalol, and flecainide on WT- and K801T-hERG currents

\begin{tabular}{|c|c|c|c|c|}
\hline & \multicolumn{2}{|c|}{ WT-hERG channels } & \multicolumn{2}{|c|}{ K801T-hERG channels } \\
\hline hERG & $\mathrm{IC}_{50 \text {-steadys }}(\mathrm{Hill}$ & $\mathrm{IC}_{50-\text { tails }}(\mathrm{Hill}$ & $\mathrm{IC}_{50 \text {-steadys }}(\mathrm{Hill}$ & $\mathrm{IC}_{50-\text { tails }}(\mathrm{Hill}$ \\
\hline blockers & coefficient $)(\mu \mathrm{M})$ & coefficient $)(\mu \mathrm{M})$ & coefficient $)(\mu \mathrm{M})$ & coefficient $)(\mu \mathrm{M})$ \\
\hline quinidine & $56.26(0.96)$ & $51.23(0.98)$ & $129.25(1.15)$ & $121.29(1.12)$ \\
\hline disopyramide & $68.59(0.96)$ & $66.12(0.96)$ & $167.98(1.12)$ & $158.12(1.09)$ \\
\hline sotalol & $127.85(0.92)$ & $127.85(0.92)$ & $232.51(1.02)$ & $225.28(1.06)$ \\
\hline flecainide & $145.51(0.99)$ & $145.51(0.99)$ & $307.41(1.03)$ & $295.64(1.08)$ \\
\hline
\end{tabular}

\section{In vitro electrophysiologic effects of hERG channel blockers}

The effects of four hERG channel blockers, quinidine, disopyramide, sotalol, and flecainide on WT- and K801T-hERG currents are illustrated in Fig. 6. Concentration response relationships were obtained by fitting the data with a Hill equation to yield $\mathrm{IC}_{50}$ values. For WT-hERG channels, the $\mathrm{IC}_{50 \text {-steadys }}$ are as follows (Hill coefficient in parentheses): quinidine, $56.26 \mu \mathrm{M}(0.96)$; disopyramide, $68.59 \mu \mathrm{M}(0.98)$; sotalol, $123.82 \mu \mathrm{M}(0.94)$; and flecainide, $159.51 \mu \mathrm{M}(0.96)$, and the $\mathrm{IC}_{50-\text { tails }}$ are as follows (Hill coefficient in parentheses): quinidine, $51.23 \mu \mathrm{M}$ (0.98); disopyramide, $66.12 \mu \mathrm{M}(0.96)$; sotalol, $127.85 \mu \mathrm{M}(0.92)$; and flecainide, $145.51 \mu \mathrm{M}(0.99)$. For K801T-hERG channels, the $\mathrm{IC}_{50 \text {-steadys }}$ are as follows: quinidine, 129.25 $\mu \mathrm{M}$ (1.15); disopyramide, $167.98 \mu \mathrm{M}$ (1.12); sotalol, $232.51 \mu \mathrm{M}$ (1.02); and flecainide, 307.41 $\mu \mathrm{M}$ (1.03), and the $\mathrm{IC}_{50 \text {-tails }}$ are as follows (Hill coefficient in parentheses): quinidine, 121.29 $\mu \mathrm{M}$ (1.12); disopyramide, $158.12 \mu \mathrm{M}$ (1.09); sotalol, $225.28 \mu \mathrm{M}$ (1.06); and flecainide, 295.64 $\mu \mathrm{M}$ (1.08) (Table 1).

The $\mathrm{IC}_{50}$ of the four drugs in K801T-hERG channels was significantly higher than that of WT-hERG channels, indicating the inhibition of hERG blockers on K801T-hERG channels was weaker than WT-hERG channels. All four drugs tested inhibited WT-hERG and K801T-hERG in concentration response, and the potencies of these drugs can be rank-ordered as follows: quinidine > disopyramide > sotalol > flecainide. A Hill coefficient close to 1.0 was obtained for each drug, indicating a single binding site to the hERG channel. 


\section{Cellular Physiology Cell Physiol Biochem 2018;51:1301-1312 and Biochemistry \begin{tabular}{c|c|c|} 
DOl: 10.1159/000495549 & Published online:27 November 2018 www.karger.com/cpb
\end{tabular} \\ Cheng et al.: A Heterozygous Missense hERG Mutation Associated with Early \\ Repolarization Syndrome}

\section{Discussion}

We report herein a novel mutation, K801T, in the hERG gene in a single Chinese family with ERS. Our results show that the K801T mutation leads to a significant increase in hERG current, a significant negative shift in the voltage dependence of activation, faster rate of activation and deactivation, and a positive shift in the voltage dependence of inactivation, indicating that gain-of-function mutation of hERG channel might be a genetic basis for ERS. The hERG channel blockers, including quinidine, disopyramide, sotalol and flecainide, inhibited K801T-hERG channel in concentration response.

It is important to emphasize that, although ERP is common finding in the general population, only a small subset of patients with this pattern have an increased risk for SCD. Currently, however, there are no tools available, including ECG phenotype, provocative drug testing and electrophysiological studies to improve risk stratification [13]. Interestingly, ERP and Brugada syndrome (BrS) share some common clinical traits, including the mean age of onset, male predominance, variability of the ECG alterations, and response to drugs or autonomic modulation. Genetically, loss-of-function mutations in SCN5A, CACNA1C, CACNB2 and CACNA2D, and gain-of-function mutations in KCNJ8 and hERG have been identified to be associated with BrS [14]. Given these evidences, it is highly likely that a genetic basis for ERS also exists.

The first genetic study of ERS was a case report that described a rare variant in KCNJ8, responsible for the pore forming subunit of the cardiac $\mathrm{I}_{\mathrm{K}-\mathrm{ATP}}$ channel, in a 14-year-old girl presenting with an ERP and frequent VF episodes [15]. Burashnikov et al. reported four patients with ERS, carrying loss of function mutations in the $\alpha 1$ and $\beta 2$ and $\alpha 2 \delta$ subunits of the cardiac L-type calcium channel (CACNA1C, CACNB2, and CACNA2D1) [16]. The most recent addition to the genes associated with ERS is SCN5A, the gene that encodes the $\alpha$ subunit of the cardiac sodium channel [17]. Our results identify a novel hERG mutation to produce a remarkable gain of function and provide a novel genetic basis for the ERS. The K801T mutation of hERG gene was only found in the proband and affected family members with marked ERP and was not present in a control population of 150 individuals, there might be a causal relationship between the mutation and ERS. As could be predicted from gain-offunction hERG mutations that lead to BrS, the K801T mutation associated with ERS in the family reported herein was associated with a gain-of-function.

The hERG channel has previously been linked to an increase in outward repolarizing current responsible for the short QT syndrome and $\mathrm{BrS}[18,19]$. In patients with BrS, several gain-of-function hERG mutations (G873S, W927G, N985S and R1135H) have been reported to increase the $\mathrm{I}_{\mathrm{Kr}}$ current densities and the susceptibility to all-or-none repolarization (loss-of-dome of action potentials) [18, 20]. Given the similarity of mechanisms between ERS and BrS, we proposed the mutation-induced increase in $\mathrm{I}_{\mathrm{Kr}}$ during initial activation may also contribute to the premature repolarization in patients with ERS. We next investigated whether the K801T change itself could be responsible for these effects. Electrophysiological characterization of the T801-hERG channel revealed that this channel exhibited biophysical properties quite different from those of the K801-hERG channel, with the peak tail current increasing by 1.5 folds, and voltage dependence of steady-state activation shifting by $12 \mathrm{mV}$, and time courses of deactivation accelerating by approximately twofold. Of note, the rates of activation and deactivation were also faster in T801-hERG channel than in T801-hERG channel. Despite the effects were relatively small, they still reach statistical significance. These hasten repolarization, which would translate into ERP phenotype on the surface ECG and increase the vulnerability to VF.

The K801 residue in hERG is evolutionary highly conserved from mouse and rat to human, suggesting that replacing this codon with threonine might influence the function of the hERG channel. The K801T variant is located in the C-terminus of the hERG channel, a site that plays a critical role in cardiac repolarization regulating the outward $\mathrm{K}+$ current 


\section{Cellular Physiology Cell Physiol Biochem 2018;51:1301-1312

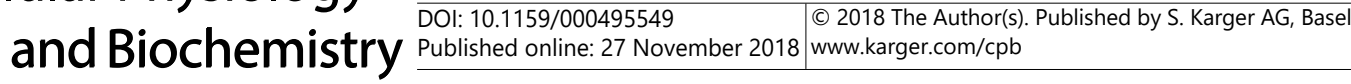 \\ Cheng et al.: A Heterozygous Missense hERG Mutation Associated with Early \\ Repolarization Syndrome}

by an interaction with the N-terminus, and delaying the channel inactivation by a knock-off mechanism [21]. In addition, the substitution of a basic lysine for a neutral threonine might also result in a change of ionic properties of the involved region of hERG channel.

Although pharmacologic therapy of ERS has not been systematically assessed, quinidine has been demonstrated to exert an ameliorative effect in preventing or quieting arrhythmias associated with ERS [15]. In addition, The Class III antiarrhythmic drug sotalol has been found to be effective against the hERG mutation in short QT syndrome [19]. We therefore compared between WT and K801T-hERG the potency of hERG current inhibition for class I and class III antiarrhythmic drugs under conventional voltage clamp. In our experiment, quinidine and disopyramide exhibit greater blocking potency than sotalol and flecainide on both WT and K801T-hERG channel, indicating quinidine and disopyramide may be worthy of investigation as a potential treatment for ERS. We also found the four drugs tested exhibited weaker inhibition potency on K801T-hERG channels than on WT-hERG channels, and this suggests the K801T missense mutation could decrease the affinity and interaction of the channel for drugs with class I and class III antiarrhythmic action. Previous studies demonstrated many antiarrhythmic drugs bind to their receptors on hERG channels in the open state, which is thought to be stabilized by inactivation of the channel [22]. The shift of K801T hERG channel inactivation to more positive voltages has been suggested to be the explanation for the decreased affinity of these channels to class I and class III antiarrhythmic drugs [19].

\section{Conclusion}

In conclusion, we investigated a rare ERS family with a high incidence of SCD, in which we found a pathogenic mutation in hERG (p.K801T) with gain-of-function. The targeted mutation screening might provide a promising prognostic tool for risk stratification of patients with ERP. Quinidine and disopyramide, in our present study, could improve the function of K801T-hERG mutant channel. However, further clinical trials are needed to elucidate the therapeutic feasibility to human.

\section{Abbreviations}

ERP (Early repolarization pattern); ERS (Early repolarization syndrome); hERG (The human Ether-à-go-go-Related Gene, also known as gene KCNH2); ECG (electrocardiogram ); VF (ventricular fibrillation); VT (ventricular tachycardia); SCD (sudden cardiac death); HEK293 (Human embryonic kidney 293); PCR (polymerase chain reaction); BrS (Brugada syndrome).

\section{Acknowledgements}

This work was supported by National Natural Science Foundation of China (No. 81370285), Guangdong Province Science and Technology Program (No. 2012B031800091), and Guangzhou City Science and Technology Program (No. 201508020057) to Dr. Wu; and National Natural Science Foundation of China for Young Scholar (No. 81600260), Natural Science Foundation of Guangdong Province (No. 2016A030313210), Project of Guangdong Province Science and Technology Plan (No. 2017A020215174), Medical and health project of Huangpu district (NO. 201607), the Fundamental Research Funds for the Central Universities in Sun Yat-Sen University (18ykpy08), and Project of Kelin new star of the First Affiliated Hospital of Sun Yat-Sen University (No. Y50186) to Dr. Cheng. 


\section{Cellular Physiology Cell Physiol Biochem 2018;51:1301-1312

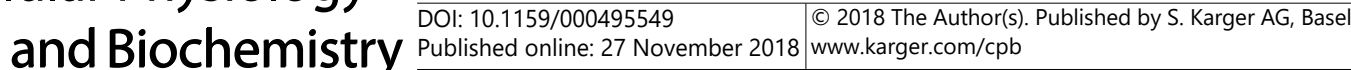 \\ Cheng et al.: A Heterozygous Missense hERG Mutation Associated with Early \\ Repolarization Syndrome}

\section{Disclosure Statement}

The authors have no competing interest.

\section{References}

1 Macfarlane PW, Antzelevitch C, Haissaguerre M, Huikuri HV, Potse M, Rosso R, Sacher F, Tikkanen JT, Wellens H, Yan GX: The Early Repolarization Pattern: A Consensus Paper. J Am Coll Cardiol 2015;66:470477.

-2 Tikkanen JT, Anttonen 0, Junttila MJ, Aro AL, Kerola T, Rissanen HA, Reunanen A, Huikuri HV: Long-term outcome associated with early repolarization on electrocardiography. N Engl J Med 2009;361:2529-2537.

-3 Cheng YJ, Mei WY, Chen XM, Liu LJ, Zheng DD, Ji CC, Tang K, Wu SH: Long-term prognosis associated with early repolarisation pattern in Chinese population with atherosclerotic risk factors. Heart 2017;103:910916.

4 Haissaguerre M, Derval N, Sacher F, Jesel L, Deisenhofer I, de Roy L, Pasquie JL, Nogami A, Babuty D, YliMayry S, De Chillou C, Scanu P, Mabo P, Matsuo S, Probst V, Le Scouarnec S, Defaye P, Schlaepfer J, Rostock T, Lacroix D et al.:. Sudden cardiac arrest associated with early repolarization. N Engl J Med 2008;358:20162023.

5 Wu SH, Lin XX, Cheng YJ, Qiang CC, Zhang J: Early repolarization pattern and risk for arrhythmia death: a meta-analysis. J Am Coll Cardiol 2013;61:645-650.

6 Cheng YJ, Lin XX, Ji CC, Chen XM, Liu LJ, Tang K, Wu SH: Role of Early Repolarization Pattern in Increasing Risk of Death. J Am Heart Assoc 2016;5.

7 Priori SG, Blomstrom-Lundqvist C: 2015 European Society of Cardiology Guidelines for the management of patients with ventricular arrhythmias and the prevention of sudden cardiac death summarized by cochairs. Eur Heart J 2015;36:2757-2759.

-8 Nunn LM, Bhar-Amato J, Lowe MD, Macfarlane PW, Rogers P, McKenna WJ, Elliott PM, Lambiase PD: Prevalence of J-point elevation in sudden arrhythmic death syndrome families. J Am Coll Cardiol 2011;58:286-290.

-9 Patton KK, Ellinor PT, Ezekowitz M, Kowey P, Lubitz SA, Perez M, Piccini J, Turakhia M, Wang P, Viskin S: Electrocardiographic Early Repolarization: A Scientific Statement From the American Heart Association. Circulation 2016;133:1520-1529.

10 Vandenberg JI, Perry MD, Perrin MJ, Mann SA, Ke Y, Hill AP: hERG K(+) channels: structure, function, and clinical significance. Physiol Rev 2012;92:1393-1478.

11 Bezzina CR, Verkerk AO, Busjahn A, Jeron A, Erdmann J, Koopmann TT, Bhuiyan ZA, Wilders R, Mannens MM, Tan HL, Luft FC, Schunkert H, Wilde AA: A common polymorphism in KCNH2 (HERG) hastens cardiac repolarization. Cardiovasc Res 2003;59:27-36.

12 Volberg WA, Koci BJ, Su W, Lin J, Zhou J: Blockade of human cardiac potassium channel human ether-a-gogo-related gene (HERG) by macrolide antibiotics. J Pharmacol Exp Ther 2002;302:320-327.

13 Mahida S, Derval N, Sacher F, Leenhardt A, Deisenhofer I, Babuty D, Schlapfer J, de Roy L, Frank R, Yli-Mayry S, Mabo P, Rostock T, Nogami A, Pasquie JL, de Chillou C, Kautzner J, Jesel L, Maury P, Berte B, Yamashita S et al.: Role of electrophysiological studies in predicting risk of ventricular arrhythmia in early repolarization syndrome. J Am Coll Cardiol 2015;65:151-159.

14 Antzelevitch C, Yan GX, Ackerman MJ, Borggrefe M, Corrado D, Guo J, Gussak I, Hasdemir C, Horie M, Huikuri H, Ma C, Morita H, Nam GB, Sacher F, Shimizu W, Viskin S, Wilde AA: J-Wave syndromes expert consensus conference report: Emerging concepts and gaps in knowledge. Heart Rhythm 2016;13:e295-324.

15 Haissaguerre M, Chatel S, Sacher F, Weerasooriya R, Probst V, Loussouarn G, Horlitz M, Liersch R, SchulzeBahr E, Wilde A, Kaab S, Koster J, Rudy Y, Le Marec H, Schott JJ: Ventricular fibrillation with prominent early repolarization associated with a rare variant of KCNJ8/KATP channel. J Cardiovasc Electrophysiol 2009;20:93-98.

-16 Burashnikov E, Pfeiffer R, Barajas-Martinez H, Delpon E, Hu D, Desai M, Borggrefe M, Haissaguerre M, Kanter R, Pollevick GD, Guerchicoff A, Laino R, Marieb M, Nademanee K, Nam GB, Robles R, Schimpf R, Stapleton DD, Viskin S, Winters S et al.: Mutations in the cardiac L-type calcium channel associated with inherited J-wave syndromes and sudden cardiac death. Heart Rhythm 2010;7:1872-1882. 


\section{Cellular Physiology Cell Physiol Biochem 2018;51:1301-1312}

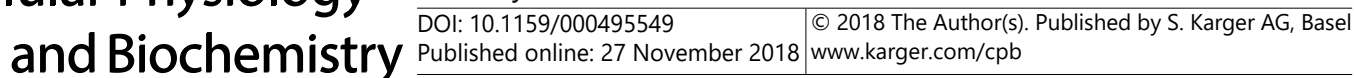

17 Guo Q Ren L, Chen X, Hou C, Chu J, Pu J, Zhang S: A novel mutation in the SCN5A gene contributes to arrhythmogenic characteristics of early repolarization syndrome. Int J Mol Med 2016;37:727-733.

18 Verkerk AO, Wilders R, Schulze-Bahr E, Beekman L, Bhuiyan ZA, Bertrand J, Eckardt L, Lin D, Borggrefe M, Breithardt G, Mannens MM, Tan HL, Wilde AA, Bezzina CR: Role of sequence variations in the human ethera-go-go-related gene (HERG, KCNH2) in the Brugada syndrome. Cardiovasc Res 2005;68:441-453.

19 Brugada R, Hong K, Dumaine R, Cordeiro J, Gaita F, Borggrefe M, Menendez TM, Brugada J, Pollevick GD, Wolpert C, Burashnikov E, Matsuo K, Wu YS, Guerchicoff A, Bianchi F, Giustetto C, Schimpf R, Brugada P, Antzelevitch C: Sudden death associated with short-QT syndrome linked to mutations in HERG. Circulation 2004;109:30-35.

20 Wilders R, Verkerk AO: Role of the R1135H KCNH2 mutation in Brugada syndrome. Int J Cardiol 2010;144:149-151.

-21 Aydar E, Palmer C: Functional characterization of the C-terminus of the human ether-a-go-go-related gene K(+) channel (HERG). J Physiol 2001;534:1-14.

-22 Numaguchi H, Mullins FM, Johnson JJ, Johns DC, Po SS, Yang IC, Tomaselli GF, Balser JR: Probing the interaction between inactivation gating and Dd-sotalol block of HERG. Circ Res 2000;87:1012-1018. 